\title{
Electronic and Magnetic Structures of $\mathrm{Ba}_{2} \mathrm{MReO}_{6}(M=\mathrm{Mn}, \mathrm{Fe}, \mathrm{Co}$, and Ni)
}

\author{
J. H. Park, S. K. Kwon, and B. I. Min* \\ Department of Physics, Pohang University of Science and Technology, Pohang 790-784, Korea
}

(Received 6 June 2007)

\begin{abstract}
Electronic structures of ordered double perovskites $\mathrm{Ba}_{2} \mathrm{MReO}_{6}(\mathrm{M}=\mathrm{Mn}, \mathrm{Fe}, \mathrm{Co}$, and $\mathrm{Ni})$ are investigated by using the linearized muffin-tin orbitals band method in the local spin-density approximation (LSDA) and the LSDA $+U$ method. The half-metallic ferrimagnetic ground states are obtained for $M=\mathrm{Fe}$ and $\mathrm{Ni}$ in the LSDA $+U$, whereas the insulating ground state is obtained for $M=M n$ in the LSDA+ $U$ incorporating the spinorbit interaction. For $M=\mathrm{Co}$, the antiferromagnetic ground state is stabilized in the LSDA+U by invoking the structural distortion.
\end{abstract}

Keywords : electronic structure, double-perovskites, half-metal, spin-orbit interaction

\section{Introduction}

The colossal magnetoresistance (CMR) phenomenon has been firstly found in manganese oxides with perovskite structure [1] and also its derivative structures. However, the low operating temperatures $\left(T_{\mathrm{C}}<250 \mathrm{~K}\right)$ in these materials hinder the practical applications. Therefore, the room temperature CMR phenomena in ordered double perovskites $\mathrm{Sr}_{2} \mathrm{Fe}^{\prime} \mathrm{O}_{6}\left(M^{\prime}=\mathrm{Mo}\right.$ and $\left.\mathrm{Re}\right)$ are essentially interesting to overcome such a problem [2-13].

The replacement of the $\mathrm{B}$ cation in the perovskite $\mathrm{ABO}_{3}$ structure by two different kinds of cations results in the ordered double perovskite structure as $A_{2} B^{\prime} B^{\prime \prime} \mathrm{O}_{6}$. This structure still retains the over-all cubic symmetry and the octahedral environment at the $B$ cation sites.

Compound of the type $A_{2}^{2+} \mathrm{FeMoO}_{6}$ with the ordered perovskite structure have been reported with $A=\mathrm{Ca}, \mathrm{Sr}$, and $\mathrm{Ba}[4,5]$. For $A=\mathrm{Sr}$ and $\mathrm{Ba}$, the tunneling magnetoresistance (TMR) phenomena is observed, but not for $A=\mathrm{Ca}$ in the ceramic phase. As changing the $A$ cation from $\mathrm{Ba}$ to $\mathrm{Sr}$ and $\mathrm{Ca}$, the cubic symmetry turns into the tetragonal and the orthorhombic symmetry. Ito et al. [13] have reported the structural and magnetic properties of $\mathrm{Sr}_{2} M \mathrm{MoO}_{6}(M=\mathrm{Mn}, \mathrm{Fe}, \mathrm{Co})$ : antiferromagnetic semiconductor for $M=\mathrm{Mn}$, Co and ferrimagnetic metal for $M=\mathrm{Fe}$.

In contrast to active researches done on the Mo-based double perovskite, studies on Re-based compounds are relatively scarce [14-17]. Sleight et al. [14] investigated magnetic and electronic characteristics of the ordered double perovskite $\mathrm{Ba}_{2} M \mathrm{ReO}_{6}(M=\mathrm{Mn}, \mathrm{Fe}, \mathrm{Co}$, and Ni). The magnetic features of them are somewhat similar to the Mo-based double perovskites: ferrimagnetic for $M=$ $\mathrm{Mn}, \mathrm{Fe}, \mathrm{Ni}$, and antiferromagnetic for $M=\mathrm{Co}$. These Rebased double perovskites supply strong evidence for the half-metallic characteristics of integer magnetic moments. The measured magnetic moments are close to integer values, $3.91 \mu_{B}, 3.06 \mu_{B}$, and $1.03 \mu_{B}$ for $M=\mathrm{Mn}, \mathrm{Fe}$ and Co, respectively.

Although both $\mathrm{Sr}_{2} \mathrm{FeMoO}_{6}$ and $\mathrm{Sr}_{2} \mathrm{FeReO}_{6}$ exhibit seemingly similar electronic and magnetic characteristics, it should be noted the differences of electronic states of Mo $4 d$ and Re $5 d$ ions. A major difference between Mo and Re oxides is electronic configuration: $\mathrm{Mo}^{5+}$ has $4 d^{1}$ electron configuration, whereas $\mathrm{Re}^{5+}$ has $5 d^{2}$. Hence the conduction carrier density of Re system is expected twice larger than that of Mo system, while the localized spins of Fe $3 d$ electrons remain similarly in both oxides. This will have an influence on the magnetotransport behavior, in view of the fact that the low-field MR in ferromagnetic metals depend on the charge-carrier density [18]. Further, the spin-orbit (SO) interaction in $\mathrm{Re}$ ion would be important to produce the sizable orbital magnetic moment of $\operatorname{Re}$ ion $[19,20]$.

*Corresponding author: Tel: +82-54-279-2074,

Fax: +82-54-278-3099, e-mail: bimin@ postech.ac.kr 


\section{Computational Method}

The linearized muffin-tin orbitals (LMTO) band structure calculations have been performed for cubic double perovskites $\mathrm{BaMReO}_{6}(M=\mathrm{Mn}, \mathrm{Fe}, \mathrm{Co}$, and $\mathrm{Ni})$. The employed experimental lattice constants are 8.181 $\AA$, $8.050 \AA$, 8.078 $\AA$, and $8.053 \AA$ for $M=\mathrm{Mn}, \mathrm{Fe}, \mathrm{Co}$, and $\mathrm{Ni}$, respectively. The density of states (DOS) were obtained by using both the local spin-density approximation (LSDA) and the LSDA $+U$ including the SO interaction [21]. The on-site Coulomb interactions $U$ and the exchange interaction $J$ parameters in the LSDA $+U$ calculations are $U=3 \sim 5 \mathrm{eV}$ for $\mathrm{Mn}, \mathrm{Fe}, \mathrm{Co}, \mathrm{Ni}$, and $2.0 \mathrm{eV}$ for $\mathrm{Re}$, respectively, and $J=0.89 \mathrm{eV}$ for all the cases.

\section{Results}

\section{1. $\mathrm{Ba}_{2} \mathrm{MnReO}_{6}$}

The LSDA electronic structure calculation produces the ferrimagnetic metallic state for $\mathrm{Ba}_{2} \mathrm{MnReO}_{6}$ (Fig. 1(a)). Spins of $\mathrm{Mn}$ and Re ions polarize antiferromagnetically, with the magnetic moment of $3.62 \mu_{B}$ and $-1.83 \mu_{B}$, respectively. The metallic result from the LSDA, however, does not agree with the insulating ground state in experiments. Therefore, one has to invoke some other mechanism to yield an insulating ground state. One possible mechanism is the Coulomb correlation effect. However, the LSDA $+U$ method also gives the halfmetallic ground state for $\mathrm{Ba}_{2} \mathrm{MnReO}_{6}$.

Hence we performed the LSDA $+U+\mathrm{SO}$ including the $\mathrm{SO}$ interaction. The DOS including the SO interaction shows a semiconducting ground state with a deep valley near the Fermi level $\left(E_{F}\right)$ in agreement with the experiment (Fig. 1(b)). The spin and orbital magnetic moments at Re site are $-1.07 \mu_{B}$ and $0.33 \mu_{B}$, respectively. The total magnetic moment per formula unit (f.u.) is $4.38 \mu_{B}$ with the spin contribution of $4.01 \mu_{B}$ and the orbital contribution of $0.37 \mu_{B}$. The localized states near $E_{F}$ correspond to the hybridized states mostly of $\operatorname{Re} t_{2 g}$ and $\mathrm{O} 2 p$ character.

\section{2. $\mathrm{Ba}_{2} \mathrm{FeReO}_{6}$}

The LSDA electronic structure calculation yields the ferrimagnetic half-metallic ground state for $\mathrm{Ba}_{2} \mathrm{FeReO}_{6}$ at the experimental lattice constant of $a=8.050 \AA$ (Fig. $1(\mathrm{c})$ ). The half-metallic nature from the LSDA is consistent with the experimental result of the metallic state for $\mathrm{Ba}_{2} \mathrm{FeReO}_{6}$. The DOS of Fig. 1(c) indicates that the exchange splittings $\Delta_{\mathrm{ex}} \cong 3-4 \mathrm{eV}$ at $\mathrm{Fe}$ and Re sites are larger than the crystal field splittings $10 D q \cong 1 \mathrm{eV}$. Hence the high spin states are favorable at both sites: the majority spin bands $\left(t_{2 g}\right.$ and $\left.e_{g}\right)$ at Fe are fully occupied

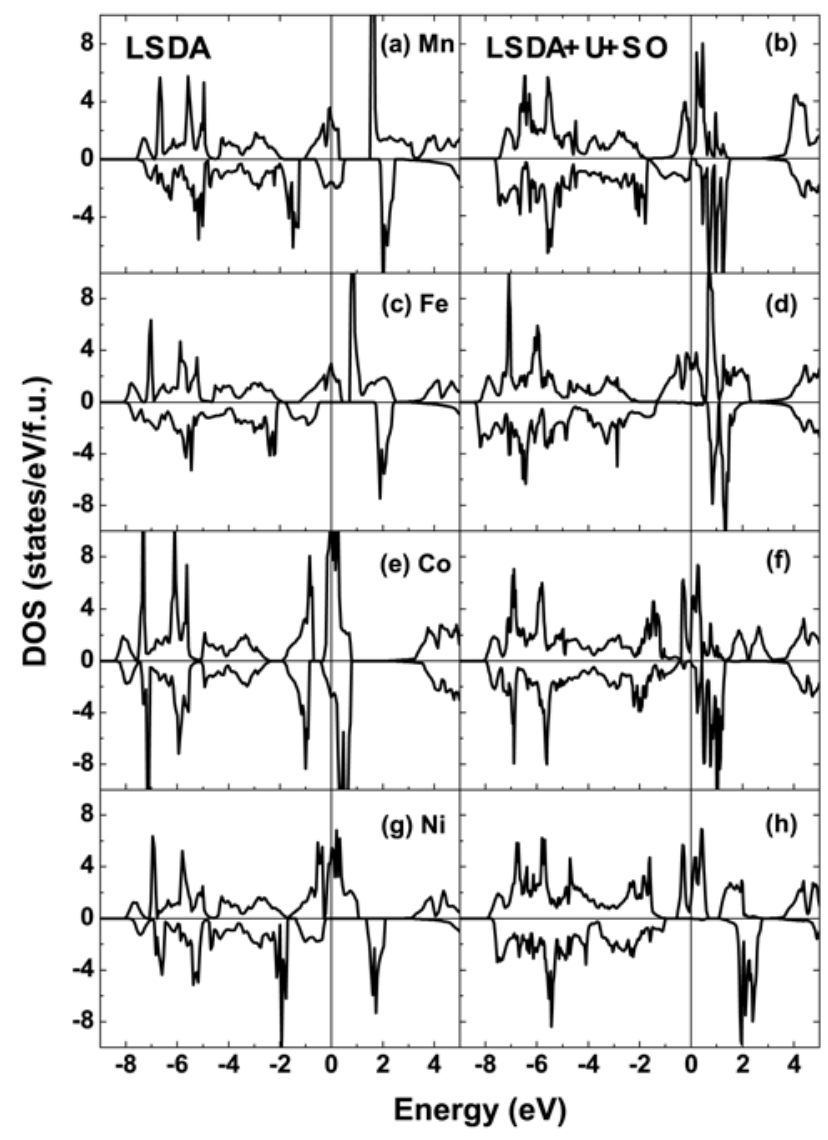

Fig. 1. The spin-polarized DOS of $\mathrm{Ba}_{2} M \mathrm{ReO}_{6}(\mathrm{M}=\mathrm{Mn}, \mathrm{Fe}$, $\mathrm{Co}, \mathrm{Ni}$ ) within the LSDA (Left) and the LSDA $+U+\mathrm{SO}$ (Right). The Fermi energy is chosen at $0.0 \mathrm{eV}$.

and the minority spin bands are partially occupied, and the majority bands at Re are empty and the minority spin bands are partially occupied. Therefore, one can expect that the spin-polarized tunneling between Fe $t_{2 g}$ and Re $t_{2 g}$ states near $E_{F}$ becomes much more effective to yield the large MR ratio.

We also performed the $\mathrm{LSDA}+U$ including the SO interaction, and the spin and orbital magnetic moments at Re are $-1.57 \mu_{\mathrm{B}}$ and $0.35 \mu_{\mathrm{B}}$, respectively. However, the electronic feature does not change much from that without the SO interaction.

\section{3. $\mathrm{Ba}_{2} \mathrm{CoReO}_{6}$}

The ferrimagnetic metallic ground state is obtained in the LSDA at the experimental lattice constant $a=8.078 \AA$ (Fig. 1(e)). In $\mathrm{BaCoReO}_{6}$, the exchange splitting at $\mathrm{Co}$ site is negligible while that at Re site is $\sim 0.5 \mathrm{eV}$, which are much smaller than the crystal field splittings of $\sim 1.2$ $\mathrm{eV}$ at $\mathrm{Co}$ and $\sim 4 \mathrm{eV}$ at $\mathrm{Re}$, and so results in the low spin state. The total energy is lower by $0.01 \mathrm{Ry} / \mathrm{f}$.u in the low spin state than in the high spin state. In addition, the 
ferromagnetic state, where the spin directions of Co and Re are parallel, is only a bit higher in the total energy by $\sim 0.01$ Ry/f.u. than the ferrimagnetic ground state. However, the experimental ground state of $\mathrm{Ba}_{2} \mathrm{CoReO}_{6}$ is semiconducting, which is different from metallic in the LSDA.

The LSDA $+U$ calculation gives the ferromagnetic ground state for $\mathrm{Ba}_{2} \mathrm{CoReO}_{6}$, which does not seem to agree with the experiment [14]. Hence we also performed the LSDA $+U$ including the SO interaction. The electronic feature, however, does not change much from that without the SO interaction. To resolve this problem, we examined a lattice distortion effect. We assumed a tetragonal structure of $a / c=0.70$ with the same volume as the cubic structure. Interestingly, the LSDA $+U$ calculation for the tetragonal $\mathrm{Ba}_{2} \mathrm{CoReO}_{6}$ yields the zero total magnetic moment per f.u. with compensated local magnetic moments of $1 \mu_{\mathrm{B}}$ at $\mathrm{Co}$ and $-1 \mu_{\mathrm{B}}$ at Re in agreement with the experimental data [14]. That is, a structural transition from cubic to tetragonal phase results in the magnetic state transition from ferromagnetic to antiferromagnetic.

\section{4. $\mathrm{Ba}_{2} \mathrm{NiReO}_{6}$}

For $\mathrm{Ba}_{2} \mathrm{NiReO}_{6}$, the LSDA calculation yields the ferromagnetic ground state at the experimental lattice constant $a=8.053 \AA$. The ferrimagnetic spin state is slightly higher in the total energy by 0.005 Ry/f.u. than that of the ferromagnetic state. However, the total magnetic moment of $3.0 \mu_{\mathrm{B}}$ in the ferromagnetic state is too larger than the experimental moment of $1.0 \mu_{\mathrm{B}}$ f.u. [14]. Therefore we apply the LSDA $+U$ method to $\mathrm{Ba}_{2} \mathrm{NiReO}_{6}$ and obtained a correct ferrimagnetic state with the magnetic moment of $1.0 \mu_{\mathrm{B}}$ in agreement with the experiment [14]. The states near $E_{F}$ are mainly from $\operatorname{Re} t_{2 g}$ and the contribution from Ni $3 d$ is negligible (Fig. 1(h)). Therefore, it is hard for carriers to hop between $\mathrm{Ni}$ and Re directly.

\section{Conclusion}

Electronic structures of double perovskite $\mathrm{Ba}_{2} \mathrm{MReO}_{6}$ were investigated by using the LSDA and the LSDA $+U$ method. The LSDA $+U$ calculations describe well the correct ferrimagnetic ground state for $\mathrm{Ba}_{2} \mathrm{MnReO}_{6}$, $\mathrm{Ba}_{2} \mathrm{FeReO}_{6}, \mathrm{Ba}_{2} \mathrm{NiReO}_{6}$ and the antiferromagnetic ground state for $\mathrm{Ba}_{2} \mathrm{CoReO}_{6}$. The $\mathrm{SO}$ interaction plays an important role in $\mathrm{Ba}_{2} \mathrm{MnReO}_{6}$ to make the system insulating. In $\mathrm{Ba}_{2} \mathrm{CoReO}_{6}$, the structural transition is invoked for the antiferromagnetic state. Also the incorporation of the $\mathrm{SO}$ interaction is essential in $\mathrm{Ba}_{2} M \mathrm{ReO}_{6}$ system to describe properly the orbital contribution of Re ions to the mag- netic moment.

\section{Acknowledgments}

This work was supported by the SRC/ERC program of MOST/KOSEF (R11-2000-071), and by the basic research program of KOSEF (R01-2006-000-10369-0).

\section{References}

[1] S. Jin, T. H. Tiefel, M. McCormack, R. A. Fastnacht, R. Ramesh, and L. H. Chen, Science 264, 413 (1994).

[2] (a) K.-I. Kobayashi, T. Kimura, H. Sawada, K. Terakura, and Y. Tokura, Nature (London) 395, 677 (1998); (b) K.I. Kobayashi, T. Kimura, H. Sawada, K. Terakura, and Y. Tokura, Phys. Rev. B 59, 11159 (1999).

[3] B. Garcia-Landa, C. Ritter, M. R. Ibarra, J. Blasco, P. A. Algarabel, R. Mahendiran, J. Garcia, Solid State Commun. 110, 435 (1999)

[4] R. P. Borges, R. M. Thomas, J. M. D. Coey, R. Suryanarayanan, L. Ben-Dor, L. Pinsard-Gaudart, and A. Revcolevschi, J. Phys.: Condens. Matter 11, L445 (1999).

[5] L. Pinsard-Gaudart, R. Suryanarayanan, A. Revcolevschi, J. Rodriguez-Carvajai, J-M. Greneche, P. A. I. Smith, R. M. Thomas, R. P. Borges, and J. M. D. Coey, J. Applied Phys. 87, 7118 (2000).

[6] Y. Moritomo, Sh. Xu, A. Machida, T. Akimoto, E. Nishibori, M. Takata, and M. Sakata, Phys. Rev. B 61, R7827 (2000).

[7] D.D. Sarma, P. Mahadevan, T. Saha-Dasgupta, S. Ray, and A. Kumar, Phys. Rev. Lett. 85, 2549 (2000).

[8] J.-S. Kang, H. Han, B. W. Lee, C. G. Olson, S. W. Han, K. H. Kim, J. I. Jeong, J. H. Park, and B. I. Min, Phys. Rev. B 64, 024429 (2001)

[9] J. -S. Kang, J. H. Kim, A. Sekiyama, S. Kasai, S. Suga, S. W. Han, K. H. Kim, T. Muro, Y. Saitoh, C. Hwang, C. G. Olson, B. J. Park, B. W. Lee, J. H. Shim, J. H. Park, and B. I. Min, Phys. Rev. B 66, 113105 (2002).

[10] J. H. Park, S. K. Kwon, and B. I. Min, Phys. Rev. B 65, 174401 (2002).

[11] J. Kim, B. J. Park, and B. W. Lee, J. Magnetics 9, 9 (2004).

[12] J. Kim, H. M. Yang, and B. W. Lee, J. Magnetics 10, 10 (2005).

[13] M. Itoh, I. Ohta, and Y. Inaguma, Mater. Sci. Eng. B 41, 55 (1996).

[14] A. W. Sleight and J. F. Weiher, J. Phys. Chem. Solids 33, 679 (1972).

[15] H. Wu, Phys. Rev. B 64, 125126 (2001).

[16] J. Herrero-Martin, G. Subias, J. Blasco, et al., J. Phys.: Condens Matter 17, 4963 (2005).

[17] J. Cheng and Z. Q. Yang, Physica Status Solidi B 243, 1151 (2006).

[18] The resistivity of $\mathrm{Ba}_{2} \mathrm{FeReO}_{6}$ is $10^{4}$ times smaller than 
that of $\mathrm{Ba}_{2} \mathrm{FeMoO}_{6}$.

[19] M. Sikora, C. Kapusta, M. Borowiec, et al., Appl. Phys. Lett. 89, 062509 (2006).

[20] C. Azimonte, J.C. Cezar, E. Granado, et al., Phys. Rev.
Lett. 98, 017204 (2007).

[21] S. K. Kwon and B. I. Min, Phys. Rev. Lett. 84, 3970 (2000). 